\title{
Subcellular localisation of pMEK has a different prognosis in locally advanced head and neck cancer treated with concomitant radiochemotherapy
}

\author{
J. Gomez-Millan ${ }^{1 *}$, B. Pajares ${ }^{2^{*+}}$, L. Perez-Villa ${ }^{3}$, A. Carnero ${ }^{4}$, M. Alvarez ${ }^{5}$, V. De Luque 2 , F. Rivas ${ }^{6,7}$, J. M. Trigo², \\ M. D. Toledo', E. Alba ${ }^{2}$ and J. A. Medina ${ }^{1}$
}

\begin{abstract}
Background: MEK1 (MAP2K1) and MEK2 (MAP2K2) are closely related dual-specificity protein kinases which function by phosphorylating both serine/threonine and tyrosine residues of their substrates ERK1 and ERK2, controlling fundamental cellular processes that include cell growth and proliferation. To investigate the prognostic significance of pMEK expression in the nucleus and cytoplasm among patients with locally advanced head and neck cancer treated with concurrent radiochemotherapy.

Methods: Immunohistochemistry was performed on the retrieved archival tissue of 96 patients to detect pMEK, p53 and Ki-67.

Results: Sixty-six percent of patients were positive for pMEK expression in the nucleus and $41 \%$ in cytoplasm. On univariate analysis, high nuclear PMEK was predictive of worse $5 y$-DFS and 5y-OS, with a trend to significance (26\% vs. $41 \%, p=0.09 ; 36 \%$ vs. $47 \%, p=0.07)$. High cytoplasmic pMEK was predictive of better 5 -y OS and 5-y DFS outcomes (61 \% vs. $27 \%, p=0.01 ; 46 \%$ vs. $22 \%, p=0.02)$. On multivariate analysis, low cytoplasmic pMEK and high nuclear pMEK predicted worse DFS and OS ( $p=0.01 ; p=0.04$ and $p=0.02 ; p=0.02$ respectively).
\end{abstract}

Conclusions: Subcellular localisation of pMEK has different prognosis in locally advanced head and neck cancer treated with radiochemotherapy.

Keywords: Head and neck cancer, Radiochemotherapy, PMEK

\section{Background}

A combination of concurrent radiotherapy and chemotherapy (RCT) is the standard treatment for locally advanced head and neck cancer (LAHNC) [1]. However, despite the intensification of radiotherapy with chemotherapy, the prognosis of these patients is still poor and involves a considerable increase in toxic effects [2]. Thus, in these patients, it is crucial to investigate new

\footnotetext{
* Correspondence: jaimegomezmillan@gmail.com; bellapajares@yahoo.es ${ }^{\dagger}$ Equal contributors

'Radiation Oncology Department, Hospital Universitario Virgen de la Victoria, Campus Teatinos s/n, 29010 Malaga, Spain

${ }^{2}$ Medical Oncology Department, Hospital Universitario Virgen de la Victoria,

Campus Teatinos s/n, 29010 Malaga, Spain

Full list of author information is available at the end of the article
}

molecular targets to improve the therapeutic ratio of treatment with RCT.

Accelerated proliferation is one of the major causes of failure in head and neck cancer treated with radiation and chemotherapy. One mechanism by which tumoural cells increase the proliferation rate in response to fractionated irradiation in head and neck cancer is EGFR phosphorylation and stimulation of the RAS/RAF/MAPK signalling pathway, a key signal transduction pathway of growth factor induced signals [3]. Within this pathway, MEK1 (MAP2K1) and MEK2 (MAP2K2) are closely related dual-specificity protein kinases that are activated by different growth factors and cytokines which function by phosphorylating both serine/threonine and tyrosine residues of their substrates ERK1 and ERK2, controlling 
fundamental cellular processes that include cell growth and proliferation $[4,5]$. There are data that support the overexpression of MEK1 as an independent biomarker of survival in other tumours such as ovarian cancer [6]. However, there are no published investigations that study the value of pMEK as a prognostic factor in head and neck cancer.

In this study, pMEK was selected as a possible prognostic factor among patients treated with RCT. We examined the expression of nuclear and cytoplasmic pMEK in tumour biopsies of LAHNC treated with RCT with regard to their response to RCT, overall survival (OS) and disease free survival (DFS).

\section{Methods}

\section{Patient data and specimen characteristics}

Between March 2000 and December 2010, 105 patients with newly diagnosed locally advanced HNSCC (stage III and IV non-metastatic), who were candidates for radical treatment, received treatment with concurrent radiochemotherapy (RCT). Of the 105 patients, 96 were fully assessable in terms of the availability of pathological specimens. Pretreatment evaluation included physical examination, endoscopy of the upper aerodigestive tract, computed tomography of the neck, and chest X-ray. In the more advanced cases (N3), computed tomography of the chest was performed. Six to eight weeks after treatment, the response was assessed under RECIST criteria. After treatment, patients underwent regular clinical and imaging examinations to assess for the occurrence of HNSCC relapse or death.

Chemotherapy was administered with Cisplatin, treatment for 70 patients was a $100 \mathrm{mg} / \mathrm{m}^{2}$ dose every 3 weeks, whereas 26 patients had $40 \mathrm{mg} / \mathrm{m}^{2}$ every week. Twenty six patients $(27 \%)$ received treatment with conventional fractionation, and the other $70(73 \%)$ were treated with accelerated fractionation with concomitant boost. Conventional fractionation was administered daily in 2 Gy per fraction, 5 days a week, to 70 Gy in 35 fractions over 7 weeks. Accelerated fractionation with concomitant boost was delivered daily in 1.8 Gy per fraction, 5 days a week, to 54 Gy in 30 fractions over 6 weeks to a clinical target volume encompassing the gross tumour and clinically/radiologically involved nodes along with regions of potential subclinical and microscopic disease. After 32.4 Gy, a second daily fraction of $1.5 \mathrm{~Gy}$ (with an interval of at least $6 \mathrm{~h}$ ) was delivered to a clinical target volume including gross tumour and involved nodes for a total of 1.8 Gy in 12 treatment days. The primary tumour and clinically/radiologically involved nodes received 72 Gy in 42 fractions over 6 weeks, and uninvolved nodes received 54 Gy over 6 weeks. Radiation treatment was planned with three-dimensional conformal radiotherapy. The median dose of radiotherapy was 71.4 Gy (70-74 Gy).

\section{Immunohistochemistry}

Tumour samples were collected during diagnosis. Immunohistochemistry (IHC) was performed on formalinfixed, paraffin-embedded (FFPE) tissue. Heat-induced antigen retrieval was performed using $0.05 \mathrm{~mol} / \mathrm{L}$ Tris buffer ( $\mathrm{pH}$ 10.0) heated to $95{ }^{\circ} \mathrm{C}$ in a steamer for $20 \mathrm{~min}$. Qualitative detection was performed as follows: for p53 (SP5, Ready to Use, MasterDiagnostica, Granada, Spain), Ki-67 and p16 (CINtec ${ }^{\circ}$ Histology Kit (MTM Laboratories AG, Germany), Phospho MEK1/2 (1:100 SER 217/221, Cell Signalling, Darmstadt, Germany). Slides were incubated with primary antibody and stained according to the standard EndVision Flex + (Dako, Copenhagen, Denmark) (K8012). As the chromogen, we used DAB and slides which were counterstained with hematoxylin. Sections of tumour tissue known to express the investigated antigens were used as positive controls. Negative controls were routinely carried out by substituting the primary antibody for non-specific IgG. The control sections were treated in parallel with the samples in the same run.

Immunohistochemical staining was evaluated independently by two authors, who were blinded to the clinical data. The ratio between the number of cells that expressed p16, Ki-67 (Fig. 1) and p53 (Fig. 2) and the whole number of tumour cells was calculated. For statistical purposes, accumulation or overexpression of p53 was considered present if $>10 \%$ of tumoural cells showed nuclear positivity [7]. Tumours were considered to have a high proliferative index if Ki-67 was positive in $>/=20 \%$ of the cells [8]. Expression of p16 was classified dichotomously as either p16-positive (strong, diffuse staining) or p16 negative [9]. Regarding pMEK, patients were divided considering staining intensity in absence of expression (0), low intensity $(+1)$, moderate intensity $(+2)$ and high intensity $(+3)$. The expression of pMEK was categorised as high expression (average intensity of +2 and +3 ) and low expression (average intensity of 0 and +1 ) (Fig. 3). pMEK positivity was considered when at least $5 \%$ of the cells presented a high expression of pMEK (average intensity of +2 and +3 ).

\section{Statistical Analysis}

The association between clinical and molecular characteristics and prognostic markers was compared using the chi-square test and Fisher's exact test when appropriate. The end points of interest used in this study were overall survival (OS), disease-free survival (DFS) and tumour relapse. OS was defined by the time that elapsed from first treatment until the event of death due to any cause. DFS was defined by the time that elapsed from the beginning of RCT to documented relapse, or death for any cause. 

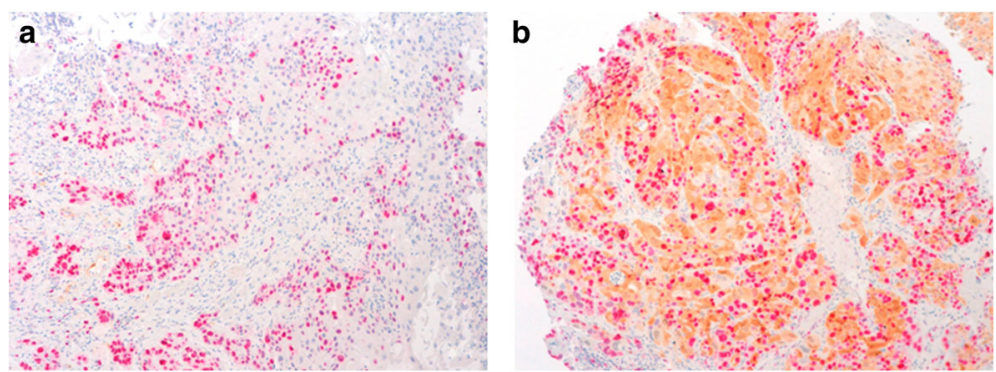

Fig. 1 Tumor cells showing negative staining for p16 and positive staining for Ki-67 (a). Positive immunostaining for p16 with some cells showing positivity for nuclear staining with Ki-67 (b)

Tumour recurrence was defined as disease recurrence any time after RCT treatment. The pattern of occurrence of the different end points was carried out by estimating Kaplan-Meier survival curves. The threshold for significance for two-side analysis was set to $p>0.05$. Multivariate survival analysis was conducted using a multivariable Cox regression model. The categorised covariates that showed a trend $(p<0.1)$ in the univariate analysis were put into a back-step multivariate Cox regression analysis. $P$-values $<0.05$ were considered significant. All analyses were carried out using $R$ and SPSS version 15.0 software.

\section{Results}

\section{Patient characteristics}

Of the 96 patients included, the vast majority in both groups were male with ECOG 0-1 performance status and stage IV disease at the time of diagnosis. Fifteen patients (18\% of the total) showed positivity for p16. Of those, two patients ( $2 \%$ of the total) were smokers. Eighty eight, $84 \%$ of the patients, showed nuclear or cytoplasmic pMEK. Nuclear pMEK staining intensity was absent $(0)$ in eight patients $(8 \%)$, weak (1+) in 31 patients (32\%), intermediate (2+) in 21 patients (22\%), and high (3+) in 36 patients (38\%). Cytoplasmic pMEK staining intensity was absent (0) in 12 patients (13\%), weak (1+) in 45 patients (47\%), intermediate (2+) in 29 patients (30\%) and high (3+) in 10 patients (10\%) (Fig. 4). Although pMEK positivity was considered when at least $5 \%$ of the cells presented a high expression of pMEK (average intensity of +2 and +3 ), $90 \%$ of the tumour samples considered as high expression presented more than $50 \%$ stained cells.

Patients were divided into low pMEK expression $(0,1+)$ or high expression $(2+, 3+)$ based on the level of staining intensity (Fig. 1). At diagnosis, 66 and $41 \%$ of the patients were categorized as having high pMEK expression in the nucleus and cytoplasm respectively. Patients with a high pMEK in cytoplasm intensity were more likely to present less advanced disease in the neck than patients with a low expression, with $77 \%$ of patients presenting a N0-1 stage in the neck compared with $23 \%$ of patients with N2-3 stage $(p=0.02)$. Characteristics of the patients are shown in Table 1.

We analysed the possible association between the site of the primary tumour and pMEK positivity and there was not a significant correlation between pMEK expression and tumour location. Thus, 49 of 71 patients (69\%) with non-oropharyngeal cancer presented nuclear pMEK positivity compared with 14 of 25 patients (56\%) with oropharynx cancer $(p=0.24)$. Moreover, 32 of 71 patients (45\%) with non-oropharyngeal cancer presented cytoplasmic pMEK positivity compared with seven of 25 patients $(28 \%)$ with oropharynx cancer $(p=0.16)$.

High nuclear pMEK expression was associated with a higher proliferation rate measured with Ki-67 expression. $56 \%$ of tumours with high nuclear pMEK presented high expression of Ki-67 and $67 \%$ of tumours with

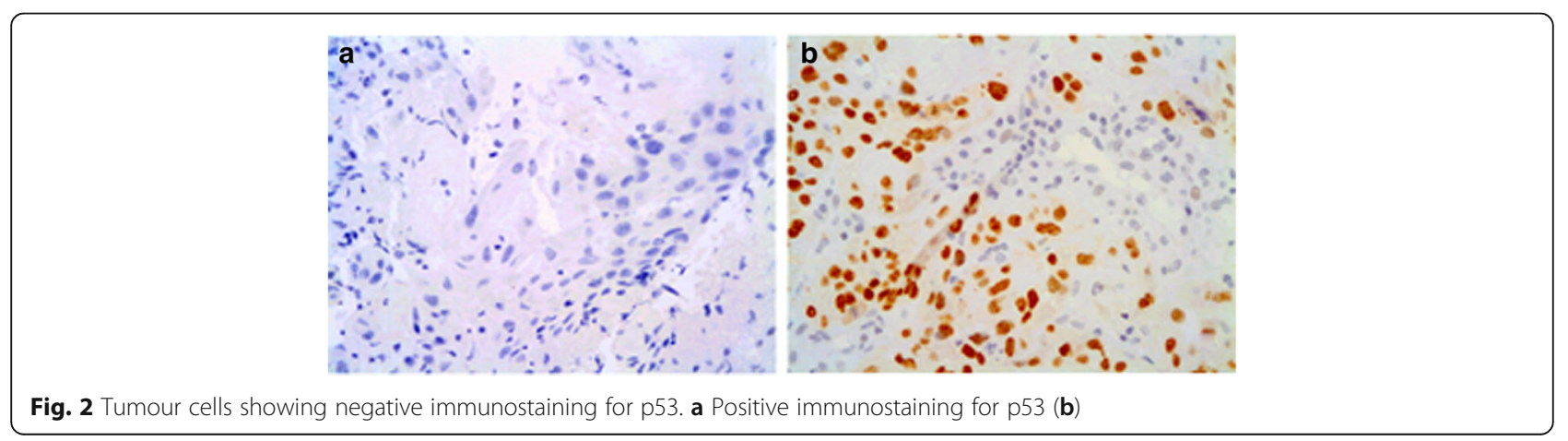




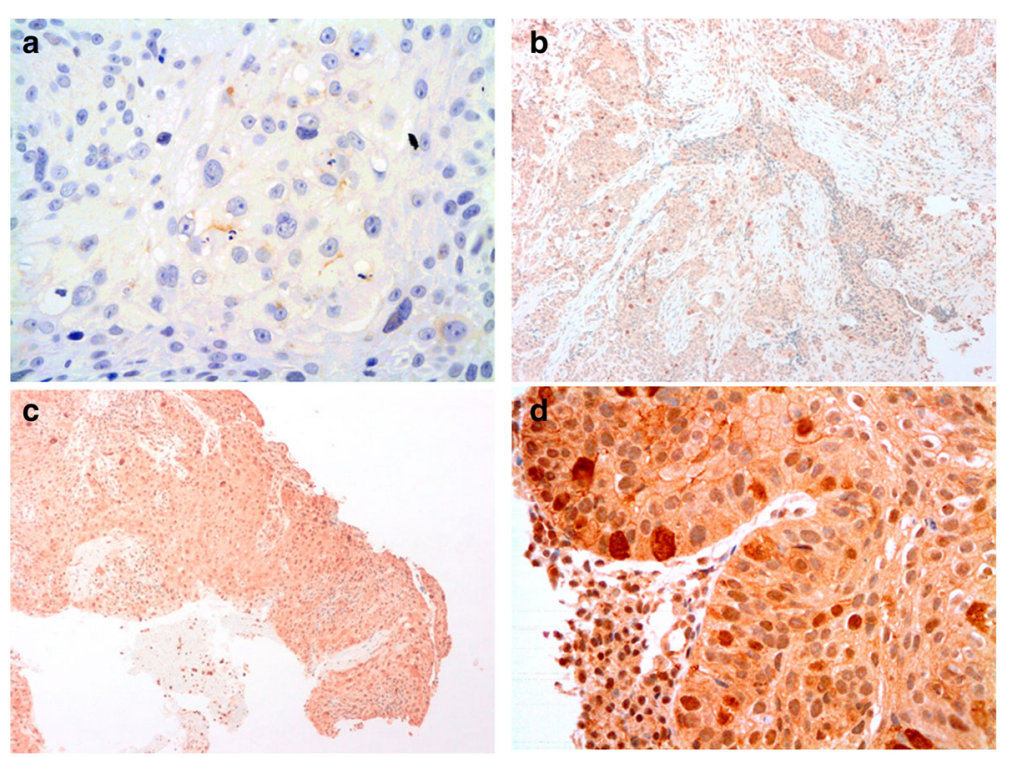

Fig. 3 Immunostaining for PMEK. a Tumours with absence of pMEK expression (0), b low expression (1),c moderate expression (2), d and high expression (3) in nucleus and cytoplasm

low nuclear pMEK presented low expression $(p=0.04)$. Furthermore, high nuclear pMEK expression was significantly associated with expression of p53: $54 \%$ of tumours with high nuclear pMEK presented expression of p53 compared with $33 \%$ in tumours with low nuclear pMEK expression $(p=0.05)$.

We analysed p16+ distribution in patients with less or equal to 50 years compared with less than 50 years old. The rate of p16+ in patients less or equal to 50 years old was $17.6 \%$ (three patients), compared with $18.5 \%$ in patients older than 50 years $(p=0.94)$.

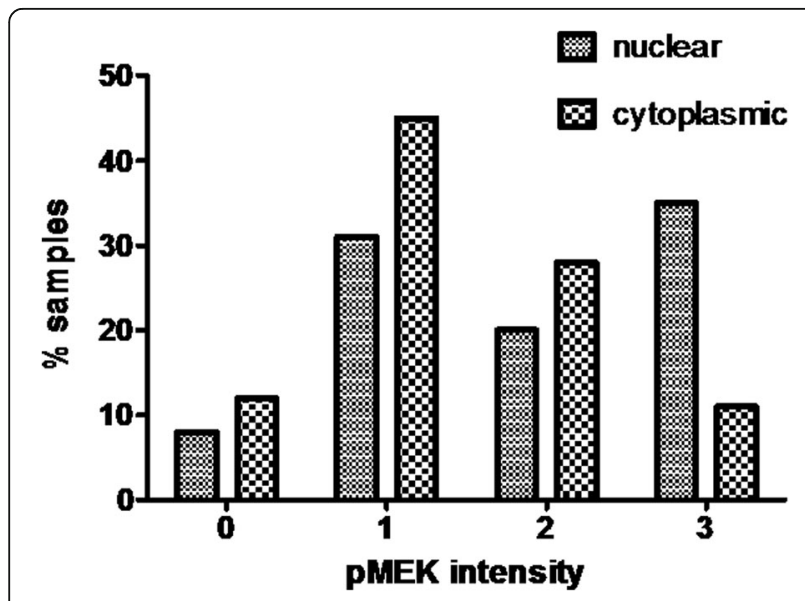

Fig. 4 Distribution of tumours with absence of pMEK expression (0), low expression (1), moderate expression (2), and high expression (3) in nucleus and cytoplasm
Subcellular localisation of pMEK localisation expression is associated with outcome

With a median follow up of 48 months, the 5-year OS and DFS were $48 \%$ (median 39 months) and $32 \%$ (median 19 months) respectively. Table 2 lists the univariate analysis for DFS and OS for various prognostic factors. Among the different clinicopathological factors studied, neck stage (N0-1 vs N2-3) was the only factor with prognostic significance, with a 5 -year OS of $49 \%$ in patients with less advanced neck disease (N0-1) compared with $27 \%$ in more advanced neck disease (N2-3) $(p=0.003)$, and a 5 -year DFS of $46 \%$ in N0-N1 patients compared with $18 \%$ in $\mathrm{N} 2-3(p=0.004)$.

To further establish the prognostic significance of pMEK, patients were stratified on the basis of nuclear and cytoplasmic pMEK expression. High nuclear pMEK was predictive of worse DFS and OS, without reaching statistical significance. On the other hand, high cytoplasmic pMEK was predictive of better OS and DFS outcomes (Fig. 5). The 5-year OS for high nuclear pMEK was $36 \%$ compared with $47 \%$ for low nuclear pMEK (HR 1.70; CI 0.95-3.02; $p=0.07$ ), and 5-year DFS was $26 \%$ compared with $41 \%$ respectively (HR 1.58; CI $0.93-2.70 ; p=0.09)$. Regarding cytoplasmic pMEK, patients with high pMEK showed a 5-year OS of $61 \%$ compared with $27 \%$ when nuclear pMEK was low (HR 0.48; CI $0.28-0.84 ; p=0.01$ ), and a DFS of $46 \%$ with high pMEK compared with $22 \%$ with low pMEK (HR 0.55; CI 0.32-0.92; $p=0.02$ ). On multivariate analysis, after adjustment for $\mathrm{T}$ stage, ECOG, and p16, advanced $\mathrm{N}$ stage, low cytoplasmic pMEK 
Table 1 Patients characteristics

\begin{tabular}{|c|c|c|c|c|c|c|}
\hline Characteristic & Low nuclear pMEK & High nuclear pMEK & $P$ value & Low cytoplasmic pMEK & High cytoplasmic pMEK & $P$ value \\
\hline $\mathrm{N}$ of patients & $33(34 \%)$ & $63(66 \%)$ & & 57 (59 \%) & 39 (41\%) & \\
\hline Age (y) & & & 0.64 & & & 0.65 \\
\hline$<50$ & $6(18 \%)$ & $14(22 \%)$ & & $11(19 \%)$ & $9(23 \%)$ & \\
\hline$>/=50$ & $27(82 \%)$ & 49 (78 \%) & & 46 (81\%) & $30(77 \%)$ & \\
\hline Sex & & & 0.5 & & & 0.01 \\
\hline Male & 31 (94%) & 61 (97 \%) & & 57 (100 \%) & 35 (90 \%) & \\
\hline Female & $2(6 \%)$ & $2(3 \%)$ & & $0(0 \%)$ & $4(10 \%)$ & \\
\hline Current smoker & & & 0.78 & & & 0.36 \\
\hline No & $2(6 \%)$ & $3(5 \%)$ & & $2(4 \%)$ & $3(8 \%)$ & \\
\hline Yes & 31 (94 \%) & 60 (95\%) & & 55 (96 \%) & 36 (92\%) & \\
\hline ECOG & & & 0.96 & & & 0.71 \\
\hline $0-1$ & 31 (94\%) & 59 (93\%) & & $53(93 \%)$ & 37 (95 \%) & \\
\hline $2-3$ & $2(6 \%)$ & $4(6 \%)$ & & $4(7 \%)$ & $2(5 \%)$ & \\
\hline Primary tumor & & & 0.24 & & & 0.14 \\
\hline Oropharynx & $22(67 \%)$ & 49 (78 \%) & & 39 (68 \%) & $32(82 \%)$ & \\
\hline Other & $11(33 \%)$ & $14(22 \%)$ & & $18(32 \%)$ & $7(18 \%)$ & \\
\hline T classification & & & 0.59 & & & 0.32 \\
\hline $\mathrm{T} 1-2$ & $3(9 \%)$ & $8(13 \%)$ & & $5(9 \%)$ & $6(15 \%)$ & \\
\hline T3-4 & 30 (90%) & 55 (38 \%) & & 52 (91%) & $33(85 \%)$ & \\
\hline N classification & & & 0.29 & & & 0.02 \\
\hline No-1 & $9(27 \%)$ & 24 (38 \%) & & 26 (46 \%) & 30 (77 \%) & \\
\hline N2-3 & $24(73 \%)$ & 39 (62 \%) & & 31 (54%) & $9(23 \%)$ & \\
\hline P16 & & & 0.45 & & & 0.93 \\
\hline Negative & 24 (86 \%) & 43 (80 \%) & & 41 (82 \%) & 26 (81 \%) & \\
\hline Positive & $4(14 \%)$ & 11 (20 \%) & & $9(18 \%)$ & $6(19 \%)$ & \\
\hline P53 & & & 0.05 & & & 0.91 \\
\hline$<10 \%$ & 22 (67 \%) & 29 (46 \%) & & 30 (53 \%) & 21 (54 \%) & \\
\hline$\geq 10 \%$ & 11 (33\%) & 34 (54 \%) & & 27 (47 \%) & $18(46 \%)$ & \\
\hline Ki-67 & & & 0.04 & & & 0.34 \\
\hline$<20 \%$ & 22 (67 \%) & 28 (44 \%) & & 32 (56 \%) & 18 (46 \%) & \\
\hline$\geq 20 \%$ & 11 (33 \%) & 35 (56 \%) & & 25 (44 \%) & 21 (54 \%) & \\
\hline
\end{tabular}

Abbreviations: ECOG Eastern Cooperative Oncology Group

and high nuclear pMEK predicted worse DFS and OS (Table 3).

\section{Discussion}

In this study we analyse the expression of pMEK in the nucleus and cytoplasm in patients with LAHNC treated with RCT. We show that patients with tumours that show moderate to high nuclear pMEK intensity present a lower OS and DFS compared with those who do not. Proliferation is one of the major causes of failure in head and neck cancer treated with radiation and chemotherapy, and the MAPK signal transduction pathway is one of the most important routes for the proliferation of head and neck cancer cells. In the MAPK pathway, MEK1 and MEK2 are the only activators of ERK2, processing inputs from multiple upstream kinases [10]. As a result, ERK1/2 activates different transcription factors and protein kinases, controlling the transcription and translation of genes that promote proliferation. Our results show that high nuclear expression is significantly associated with a higher proliferation rate measured with Ki-67 expression $(p=0.04)$. Although the negative prognostic impact of pMEK has been described in other tumours [6], to our knowledge there are no studies in the literature that investigate the prognostic role of pMEK in head and neck cancer. 
Table 2 Univariate analysis

\begin{tabular}{|c|c|c|c|c|}
\hline \multirow[b]{2}{*}{ Variable } & \multicolumn{2}{|l|}{ DFS } & \multicolumn{2}{|l|}{ OS } \\
\hline & $\mathrm{HR}(95 \% \mathrm{Cl})$ & $P$ value & $\mathrm{HR}(95 \% \mathrm{Cl})$ & $P$ value \\
\hline Age $(<50$ vs $\geq 50)$ & $1.09(0.60-1.90)$ & 0.70 & $1.24(0.73-2.12)$ & 0.43 \\
\hline Oropharynx vs non-oropharynx & $1.30(0.82-2.29)$ & 0.23 & $1.20(0.73-2.10)$ & 0.40 \\
\hline T stage (T1/2 vs T3/4) & $0.91(0.43-1.91)$ & 0.79 & $1.03(0.47-2.27)$ & 0.98 \\
\hline N stage (0-1 vs 2-3) & $2.03(1.26-3.28)$ & 0.01 & $2.11(1.20-3.50)$ & 0.01 \\
\hline ECOG performance status ( 0 vs $\geq 1$ ) & $1.50(0.87-2.47)$ & 0.14 & $1.50(0.87-2.58)$ & 0.14 \\
\hline P16 & $1.30(0.73-2.51)$ & 0.34 & $1.36(0.71-2.59)$ & 0.35 \\
\hline Ki-67 & $0.82(0.51-1.33)$ & 0.42 & $0.95(0.57-1.56)$ & 0.83 \\
\hline P53 & $0.99(0.61-1.60)$ & 0.90 & $1.10(0.67-1.82)$ & 0.67 \\
\hline Nuclear pMEK & $1.58(0.93-2.07)$ & 0.09 & $1.70(0.95-3.02)$ & 0.07 \\
\hline Cytoplasmic pMEK & $0.55(0.32-0.92)$ & 0.02 & $0.48(0.28-0.84)$ & 0.01 \\
\hline
\end{tabular}

Abbreviations: Cl confidence interval; DFS disease-free survival; ECOG Eastern Cooperative Oncology Group; HR hazard ratio; OS overall survival

Our results show that p16 is not a prognostic factor. The lower p16 positive prevalence in our patients compared with data from different meta-analysis (22-34 \% in HNSCC and $30-41 \%$ in OPSCC) [11, 12] confirms the previous results published by our group $[9,13]$ and is possibly due to the epidemiologic profile of our population, which had a high proportion of heavy tobacco users. In this study, the vast majority of our patients were current smokers (95\%), and only two patients were p16 positive and non-smokers.
Tumour suppressor protein p53 plays a role in the regulation of genes involved in cell cycle and growth arrest, apoptosis and DNA repair, maintaining genomic stability $[14,15]$. Mutation of TP53 is one of the most frequently detectable genetic alterations in HNSCC in tumours associated with tobacco and alcohol consumption [16], and this mutation generally results in inhibition of function, limitless proliferation and immortalisation [17]. Our results show that a significantly higher proportion of tumours with high nuclear PMEK presented expression of
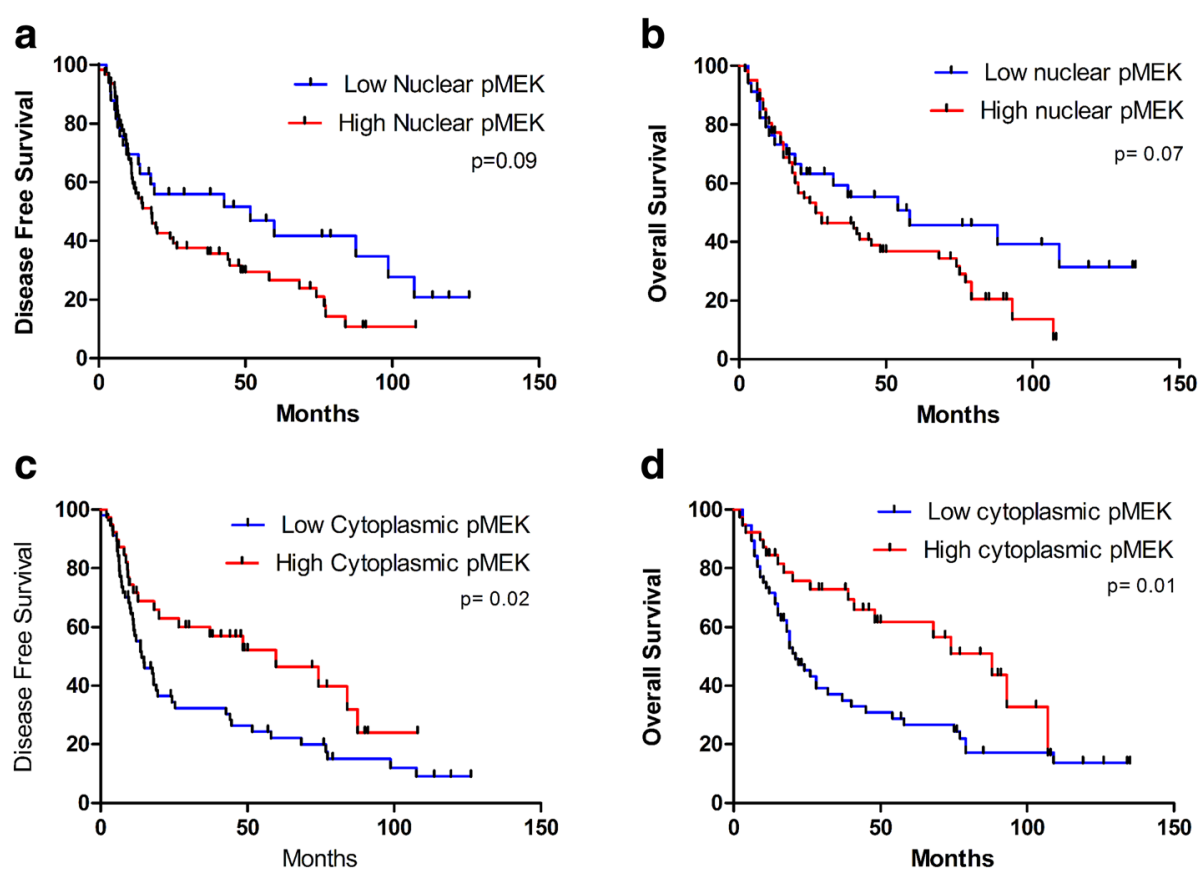

Fig. 5 Kaplan-Meier plots for disease-free survival and overall survival according to low versus high expression of pMEK in the nucleus (a, b) and cytoplasm (c, d) 
Table 3 Multivariate analysis

\begin{tabular}{|c|c|c|c|c|}
\hline \multirow[b]{2}{*}{ Variable } & \multicolumn{2}{|l|}{ DFS } & \multicolumn{2}{|l|}{ OS } \\
\hline & HR $(95 \%$ Cl) & $P$ value & $\mathrm{HR}(95 \% \mathrm{Cl})$ & $P$ value \\
\hline T stage (T1/2 vs T3/4) & $0.82(0.31-2.17)$ & 0.69 & $0.94(0.36-2.46)$ & 0.90 \\
\hline N stage (0-1 vs 2-3) & $2.34(1.33-4.04)$ & 0.03 & $2.15(1.25-3.70)$ & 0.06 \\
\hline ECOG performance status (0 vs $\geq 1$ ) & $1.74(0.93-3.26)$ & 0.08 & $1.67(0.93-2.99)$ & 0.09 \\
\hline P16 & $1.02(0.53-1.96)$ & 0.95 & $1.03(0.54-1.96)$ & 0.92 \\
\hline Nuclear pMEK & $2.21(1.14-4.30)$ & 0.02 & $2.12(1.12-3.99)$ & 0.02 \\
\hline Cytoplasmic pMEK & $0.48(0.23-0.85)$ & 0.01 & $0.53(0.29-0.97)$ & 0.04 \\
\hline
\end{tabular}

Abbreviations: Cl confidence interval; DFS disease-free survival; ECOG Eastern Cooperative Oncology Group; HR hazard ratio; OS overall survival

p53 (54 \% in high nuclear pMEK compared with $33 \%$ in low nuclear pMEK, $p=0.05)$. A link between the mutational status of p53 and activation of the Raf/Mek/Erk cascade has recently been reported in preclinical studies, showing that in the presence of Ras oncogenes and an inactive p53/p21 axis, activation of the Raf/Mek/Erk cascade leads to sustained cell proliferation $[18,19]$.

Moreover, besides regulation of cell cycle progression, the RAF/MEK/ERK signalling pathway may induce cellular responses relevant to cancer survival, such as protection from apoptosis [20, 21]. It is well known that RAF/MEK/ERK can phosphorylate BAD on S112, allowing Bcl-2 to form homodimers, generating an antiapoptotic response [22, 23]. Moreover, the association found between nuclear pMEK and p53 overexpression supports the suggestion that antiapoptotic response may well be an important mechanism of resistance to RCT in head and neck cancer.

Considering the importance that MEKK/1/2 may have in the induction of proliferation, MEK inhibitors might be potentially efficacious for the treatment of head and neck cancer. There has been interest in developing pharmacologic inhibitors of MEK as a means to blocking ERK activation in tumours with activating mutations of MEK1 or MEK2 such as ovarian, melanoma, colorectal and lung cancer [10]. The MEK inhibitor trametinib has been shown to increase overall survival in patients with BRAFV600-mutated melanoma [24] and has recently been approved for use in metastatic melanoma. In head and neck cancer, accelerated proliferation in response to ionising radiation through the activation of proliferative signalling pathways might be mitigated with MEK inhibitors. Trametinib is under investigation in combination with AKT inhibition in solid tumours including HNSCC (NCT01725100).

Secondly, we have identified cytoplasmic pMEK as a promising favourable prognostic factor for OS and DFS. Patients with cytoplasmic pMEK present superior OS and DFS compared with patients who do not express pMEK. Although pMEK rapidly translocates to the nucleus to exert its action on proliferation, it has been described that it remains in the nucleus for a short period of time due to a rapid export by the NE/Exportin system, giving rise to an apparent cytoplasmic localisation $[25,26]$ that predominates in resting cells [27]. Thus, although the functions of MEKK shuttling and cytoplasmic MEK are not fully understood [28], it might be hypothesised that the subcellular localisation of pMEK may determine different functions and may have different prognostic implications, with poor survival and an increase in proliferation markers in nuclear pMEK compared with better survival and decrease in proliferation markers in cytoplasmic pMEK. Although these finding must be confirmed, cytoplasmic pMEK may identify a subgroup of patients with a good prognosis for whom deintensification therapies might be investigated.

\section{Conclusions}

The expression of pMEK is a prognostic factor in LAHNC treated with CRT. Tumours with nuclear pMEK expression present a higher proliferation rate, showing unfavourable survival, whereas tumours with cytoplasmic expression of pMEK present a lower proliferation rate and favourable survival. Investigation of MEK inhibitors is needed to increase the prognosis of LAHNCs that are frequently not controlled with biologically non-specific treatments such as RCT. Finally, cytoplasmic pMEK expression should be explored as a prognostic biomarker in head and neck cancer.

\section{Abbreviations}

DFS: Disease free survival; ECOG: Eastern Cooperative Oncology Group; HR: Hazard ratio; LAHNC: Locally advanced head and neck cancer; OS: Overall survival; RCT: Concurrent radiotherapy and chemotherapy

\section{Acknowledgments}

This work was supported by the "Sociedad Andaluza de Cancerología" (N0:2013).

\section{Availability of supporting data}

The datasets supporting the conclusions of this article are presented in the main manuscript.

\section{Authors' contributions}

JGM and BP contributed equally to this work, they designed this study and made substantial contribution to interpretation of data. JAM and EA were the supervisors of this clinical research, revising it critically for important intellectual content. MDT and JT were responsible for gathering data. FR was responsible for the data analysis. LPV and MA were responsible for the analysis of 
immunostaining slices. VDL stained all the slices for immunohistochemistry. All authors read and approved the final manuscript.

\section{Competing interest}

The authors declare that they have no competing interest.

\section{Ethics approval and consent to participate}

This study was carried out in compliance with the Declaration of Helsinki [8]. All subjects provided written informed consent for inclusion in the study, which was approved by the CCEIBA (Comité Coordinador de Ética de la Investigación Biomédica de Andalucía) and CEls (Comités de Etica de Investigación) of Hospital Virgen de la Victoria.

\section{Author details}

'Radiation Oncology Department, Hospital Universitario Virgen de la Victoria, Campus Teatinos s/n, 29010 Malaga, Spain. ${ }^{2}$ Medical Oncology Department, Hospital Universitario Virgen de la Victoria, Campus Teatinos s/n, 29010 Malaga, Spain. ${ }^{3}$ Pathology Department, Hospital Universitario Virgen de la Victoria, Campus Teatinos s/n, 29010 Malaga, Spain. Instituto de Biomedicina de Sevilla (IBIS), Consejo superior de investigaciones científicas, Campus Universitario Virgen del Rocío, Avda, Manuel Siurot s/n, 41013 Sevilla, Spain. ${ }^{5}$ Pathology Department, Facultad de Medicina, UMA, Campus Teatinos s/n, 29010 Malaga, Spain. ${ }^{6}$ Agencia Sanitaria Costa del Sol, Unidad de Investigación, Autovia A-7, Km 187, 29063, Marbella, Málaga, Spain. ${ }^{7}$ Red Nacional de Investigación de Servicios de Salud en Enfermedades crónicas (REDISSEC), Madrid, Spain.

\section{Received: 26 April 2016 Accepted: 21 October 2016}

Published online: 28 October 2016

\section{References}

1. Blanchard P, Baujat B, Holostenco V, Bourrediem A, Baey C, Bourhis J, et al Meta-analysis of chemotherapy in head and neck cancer (MACH-NC): a comprehensive analysis by tumour site. Radiother Oncol. 2011;100(1):33-40.

2. Machtay M, Moughan J, Trotti A, Garden AS, Weber RS, Cooper JS, et al. Factors associated with severe late toxicity after concurrent chemoradiation for locally advanced head and neck cancer: an RTOG analysis. J Clin Oncol. 2008;26(21):3582-9.

3. Bussink J, Van del Kogel AJ, Kaanders JHAM. Activation of the PI3-K/AKT pathway and implications for radioresistance mechanisms in head and neck cancer. Lancet Oncol. 2008;9(3):288-96.

4. Seger R, Krebs EG. The MAPK signaling cascade. FASEB J. 1995;9(9):726-35.

5. Shaul YD, Seger R. The MEK/ERK cascade: from signaling specificity to diverse functions. Biochim Biophys Acta. 2007:1773(8):1213-26.

6. Penzvaltó Z, Lánczky A, Lénárt J, Meggyesházi N, Krenács T, Szoboszlai N, et al. MEK1 is associated with carboplatin resistance and is a prognostic biomarker in epithelial ovarian cancer. BMC Cancer. 2014;14(1):837.

7. Couture C, Raybaud-Diogene H, Tetu B, Bairati I, Murry D, Allard J, et al. p53 and $\mathrm{Ki}-65$ as markers of radioresistance in Head and Neck carcinoma. Cancer. 2002:94(3):713-22.

8. World medical association. World Medical Association Declaration of Helsinki: Ethical Principles for Medical Research Involving Human Subjects. JAMA. 2013;310(20):2191-4

9. Pajares B, Trigo JM, Toledo MD, Alvarez M, González-Hermoso C, Rueda A, et al. Differential outcome of concurrent radiotherapy plus epidermal growth factor receptor inhibitors versus radiotherapy plus cisplatin in patients with human papillomavirus-related head and neck cancer. BMC Cancer. 2013;13: 26.

10. Caunt J, Sale MJ, Smith PD, Cook S. MEK1 and MEK2 inhibitors and cancer therapy: the long and winding road. Nat Rev in cancer. 2015;15:577-92.

11. Dayyani F, Etzel CJ, Liu M, Ho CH, Lippman SM, Tsao AS. Meta-analysis of the impact of human papillomavirus (HPV) on cancer risk and overall survival in head and neck squamous cell carcinomas (HNSCC). Head Neck Oncol. 2010;29(2):15.

12. Termine N, Panzarella V, Falaschini S, Russo A, Matranga D, Lo Muzio L, et al. HPV in oral squamous cell carcinoma vs head and neck squamous cell carcinoma biopsies: a meta-analysis (1988-2007). Ann Oncol. 2008;19(10):1681-90.

13. Pajares $B$, Perez-Villa $L$, Trigo JM, Toledo MD, Alvarez $M$, Jiménez $B$, et al. Concurrent radiotherapy plus epidermal growth factor receptor inhibitors in patients with human papillomavirus-related head and neck cancer. Clin Trans Oncol. 2014;16(4):418-24.
14. Vogelstein B, Lane D, Levine AJ. Surfing the p53 network. Nature. 2000;408: 307-10.

15. Brady CA, Jiang D, Mello SS, Johnson TM, Jarvis LA, Kozak MM, et al. Distinct p53 transcriptional programs dictate acute DNA-damage responses and tumor suppression. Cell. 2011;145(4):571-83.

16. Poeta ML, Manola J, Goldwasser MA, Forastiere A, Benoit N, Califano JA, et al. TP53 mutations and survival in squamous-cell carcinoma of the head and neck. N Engl J Med. 2007;357:2552-61.

17. Morton JP, Timpson P, Karim SA, Ridgway RA, Athineos D, Doyle B, et al. Mutant p53 drives metastasis and overcomes growth arrest/senescence in pancreatic cancer. Proc Natl Acad Sci U S A. 2010;107(1):246-51.

18. Drosten M, Sum E, Lechucha C, Simón-Carrasco L, Jacob H, García-Medina R, et al. Loss of p53 induces cell proliferation via Ras-independent activation of the Raf/Mek/Erk signaling pathway. Proc Natl Acad Sci U S A. 2014;111(42): $15155-60$

19. Malumbres M, Barbacid M. RAS oncogenes: the first 30 years. Nat Rev Cancer. 2003:3(6):459-65.

20. Yoon S, Seger R. The extracellular signal-regulated kinase: multiple substrates regulate diverse cellular functions. Growth Factors. 2006:24:21-44

21. Zhao Y, Adjei A. The clinical development of MEK inhibitors. Nat Rev Clin Oncol. 2014;11(7):385-400.

22. Balmanno K, Cook SJ. Tumour cell survival signalling by the ERK1/2 pathway. Cell Death Differ. 2009;16:368-77.

23. Zha J, Harada H, Yang E, Jockel J, Korsmeyer SJ. Serine phosphorylation of death agonist BAD in response to survival factor results in binding to 14-3-3 not Bcl-XL. Cell. 1996:87:589-92.

24. Flaherty KT, Robert C, Hersey P, et al. Improved survival with MEK inhibition in BRAF-mutated melanoma. N Engl J Med. 2012;367:107-14.

25. Chuderland D, Konson A, Seger R. Identification and characterization of a general nuclear translocation signal in signaling proteins. Mol Cell. 2008;31:850-61.

26. Plotnikov A, Zehorai E, Procaccia S, Seger R. The MAPK cascades: signaling components, nuclear roles and mechanisms of nuclear translocation. Biochim Biophys Acta. 1813;2011:1619-33.

27. Jaaro $H$, Rubinfeld $H$, Hanoch $T$, Seger R. Nuclear translocation of mitogenactivated protein kinase (MEK1) in response to mitogenic stimulation. Proc Natl Acad Sci U S A. 1997;94(8):3742-7.

28. Zehorai E, Yao Z, Plotnikov A, Seger R. The subcellular localisation of MEK and ERK-A novel nuclear translocation signal (NTS) paves a way to the nucleus. Mol Cell Endocrinol. 2010;314:213-20.

\section{Submit your next manuscript to BioMed Central and we will help you at every step:}

- We accept pre-submission inquiries

- Our selector tool helps you to find the most relevant journal

- We provide round the clock customer support

- Convenient online submission

- Thorough peer review

- Inclusion in PubMed and all major indexing services

- Maximum visibility for your research

Submit your manuscript at www.biomedcentral.com/submit
C) Biomed Central 